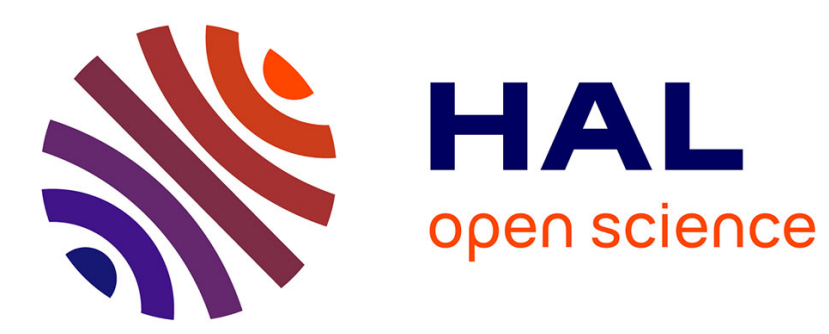

\title{
Linear interval observers under delayed measurements and delay-dependent positivity
}

Denis Efimov, Emilia Fridman, Andrey Polyakov, Wilfrid Perruquetti, Jean-Pierre Richard

\section{- To cite this version:}

Denis Efimov, Emilia Fridman, Andrey Polyakov, Wilfrid Perruquetti, Jean-Pierre Richard. Linear interval observers under delayed measurements and delay-dependent positivity. Automatica, 2016, 72, pp.123-130. 10.1016/j.automatica.2016.05.022 . hal-01312981

\section{HAL Id: hal-01312981 \\ https://inria.hal.science/hal-01312981}

Submitted on 9 May 2016

HAL is a multi-disciplinary open access archive for the deposit and dissemination of scientific research documents, whether they are published or not. The documents may come from teaching and research institutions in France or abroad, or from public or private research centers.
L'archive ouverte pluridisciplinaire HAL, est destinée au dépôt et à la diffusion de documents scientifiques de niveau recherche, publiés ou non, émanant des établissements d'enseignement et de recherche français ou étrangers, des laboratoires publics ou privés. 


\title{
Linear interval observers under delayed measurements and delay-dependent positivity
}

\author{
Denis Efimov a,b,c, Emilia Fridman $^{\mathrm{d}}$, Andrey Polyakov ${ }^{\mathrm{a}, \mathrm{b}, \mathrm{c}}$, Wilfrid Perruquetti ${ }^{\mathrm{b}, \mathrm{a}}$, \\ Jean-Pierre Richard ${ }^{\mathrm{b}, \mathrm{a}}$ \\ ${ }^{a}$ Non-A team @ Inria, Parc Scientifique de la Haute Borne, 40 av. Halley, 59650 Villeneuve d'Ascq, France \\ ${ }^{\mathrm{b}}$ CRIStAL (UMR-CNRS 9189), Ecole Centrale de Lille, BP 48, Cité Scientifique, 59651 Villeneuve-d'Ascq, France \\ ${ }^{\mathrm{c}}$ Department of Control Systems and Informatics, ITMO University, 49 Kronverkskiy av., 197101 Saint Petersburg, Russia \\ ${ }^{\mathrm{d}}$ School of Electrical Engineering, Tel-Aviv University, Tel-Aviv 69978, Israel
}

\begin{abstract}
New interval observers are designed for linear systems with time-varying delays in the case of delayed measurements. Interval observers employ positivity and stability analysis of the estimation error system, which in the case of delayed measurements should be delay-dependent. New delay-dependent conditions of positivity for linear systems with time-varying delays are introduced. Efficiency of the obtained solution is demonstrated on examples.
\end{abstract}

Key words: Interval observers, Time delay, Stability analysis

\section{Introduction}

An estimation in nonlinear delayed systems is rather complicated $[31,15]$, as well as analysis of functional differential equations [30]. Especially the observer synthesis is problematical for the cases when the model of a nonlinear delayed system contains parametric and/or signal uncertainties, or when the delay is time-varying and/or uncertain $[4,5,33]$, the frequent applications include biosystems and chemical processes. Delayed measurements usually also increase complexity of estimators, which is a case in networked systems. An observer solution for these more complex situations is highly demanded in these and many others applications. Interval or set-membership estimation is a promising framework to observation in uncertain systems $[17,19,20,24,22,27]$, when all uncertainty is included in the corresponding intervals or polytopes, and as a result the set of admissible values (an interval) for the state is provided at each instant of time.

In this work an interval observer for time-delay systems with delayed measurements is proposed. A peculiarity

\footnotetext{
* This paper was not presented at any IFAC meeting. Corresponding author is D. Efimov.
}

of an interval observer is that in addition to stability conditions, some restrictions on positivity of estimation error dynamics have to be imposed (in order to envelop the system solutions). The existing solutions in the field $[23,12,14,26]$ are based on the delay-independent conditions of positivity from $[18,2]$. Some results on interval observer design for uncertain time-varying delay can be found in $[28,12]$. The first objective of this work is to use the delay-dependent positivity conditions [13], which are based on the theory of non-oscillatory solutions for functional differential equations $[1,9]$. Next, two interval observers are designed for linear systems with delayed measurements (with time-varying delays) in the case of observable and detectable systems (with respect to [13] the present work contains new result, Theorem 12, relaxed Assumption 1, and new examples). Efficiency of the obtained interval observers is demonstrated on a benchmark example from [23] and a delayed nonlinear pendulum.

The paper is organized as follows. Some preliminaries and notation are given in Section 2. The delay-dependent positivity conditions are presented in Section 3. The interval observer design is performed for a class of linear time-delay systems (or a class of nonlinear systems in the output canonical form) with delayed measurements 
in Section 4. Examples of numerical simulation are presented in Section 5.

\section{Preliminaries}

\subsection{Notation}

- $\mathbb{R}$ is the Euclidean space $\left(\mathbb{R}_{+}=\{\tau \in \mathbb{R}: \tau \geq 0\}\right)$, $\mathcal{C}_{\tau}^{n}=C\left([-\tau, 0], \mathbb{R}^{n}\right)$ is the set of continuous maps from $[-\tau, 0]$ into $\mathbb{R}^{n}$ for $n \geq 1 ; \mathcal{C}_{\tau+}^{n}=\left\{y \in \mathcal{C}_{\tau}^{n}: y(s) \in\right.$ $\left.\mathbb{R}_{+}^{n}, s \in[-\tau, 0]\right\}$;

- $x_{t}$ is an element of $\mathcal{C}_{\tau}^{n}$ defined as $x_{t}(s)=x(t+s)$ for all $s \in[-\tau, 0]$

- $|x|$ denotes the absolute value of $x \in \mathbb{R},\|x\|_{2}$ is the Euclidean norm of a vector $x \in \mathbb{R}^{n},\|\varphi\|=$ $\sup _{t \in[-\tau, 0]}\|\varphi(t)\|_{2}$ for $\varphi \in \mathcal{C}_{\tau}^{n}$;

- for a measurable and locally essentially bounded input $u: \mathbb{R}_{+} \rightarrow \mathbb{R}^{p}$ the symbol $\|u\|_{\left(t_{0}, t_{1}\right)}$ denotes its $L_{\infty}$ norm $\|u\|_{\left[t_{0}, t_{1}\right)}=$ ess $\sup \left\{\|u(t)\|_{2}, t \in\left[t_{0}, t_{1}\right)\right\}$, the set of all such inputs $u \in \mathbb{R}^{p}$ with the property $\|u\|_{[0,+\infty)}<\infty$ will be denoted as $\mathcal{L}_{\infty}^{p}$;

- for a matrix $A \in \mathbb{R}^{n \times n}$ the vector of its eigenvalues is denoted as $\lambda(A)$;

- $I_{n}$ and $0_{n \times m}$ denote the identity and zero matrices of dimensions $n \times n$ and $n \times m$, respectively;

- $a \mathcal{R} b$ corresponds to an elementwise relation $\mathcal{R} \in\{<$ $,>, \leq, \geq\}$ ( $a$ and $b$ are vectors or matrices): for example $a<b$ (vectors) means $\forall i: a_{i}<b_{i}$; for $\phi, \varphi \in \mathcal{C}_{\tau}$ the relation $\phi \mathcal{R} \varphi$ has to be understood elementwise for whole domain of definition of the functions, i.e. $\phi(s) \mathcal{R} \varphi(s)$ for all $s \in[-\tau, 0]$;

- for a symmetric matrix $\Upsilon$, the relation $\Upsilon \prec 0(\Upsilon \preceq 0)$ means that the matrix is negative (semi) definite.

\subsection{Delay-independent conditions of positivity}

Consider a time-invariant linear system with timevarying delay:

$$
\begin{gathered}
\dot{x}(t)=A_{0} x(t)-A_{1} x(t-\tau(t))+b(t), t \geq 0, \\
x(\theta)=\phi(\theta) \text { for }-\bar{\tau} \leq \theta \leq 0, \phi \in \mathcal{C}_{\tau}^{n},
\end{gathered}
$$

where $x(t) \in \mathbb{R}^{n}, x_{t} \in \mathcal{C}_{\bar{\tau}}^{n}$ is the state function; $\tau: \mathbb{R}_{+} \rightarrow$ $[-\bar{\tau}, 0]$ is the time-varying delay, a Lebesgue measurable function of time, $\bar{\tau} \in \mathbb{R}_{+}$is the maximum delay; $b \in \mathcal{L}_{\infty}^{n}$ is the input; the constant matrices $A_{0}$ and $A_{1}$ have appropriate dimensions. The matrix $A_{0}$ is called Metzler if all its off-diagonal elements are nonnegative. The system (1) is called positive if for $x_{0} \geq 0$ it has the corresponding solution $x(t) \geq 0$ for all $t \geq 0$.

Lemma 1 [18,2] The system (1) is positive iff $A_{0}$ is Metzler, $A_{1} \leq 0$ and $b(t) \geq 0$ for all $t \geq 0$. A positive system (1) is asymptotically stable for $b(t) \equiv 0$ for all $\bar{\tau} \in \mathbb{R}_{+}$ iff there are $p, q \in \mathbb{R}_{+}^{n}(p>0$ and $q>0)$ such that

$$
p^{T}\left[A_{0}-A_{1}\right]+q^{T}=0 .
$$

Under conditions of the above lemma the system has bounded solutions for $b \in \mathcal{L}_{\infty}^{n}$. Note that for linear timeinvariant systems the conditions of positive invariance of polyhedral sets have been similarly given in [8], as well as conditions of asymptotic stability in the nonlinear case have been considered in $[6,7,3]$.

\section{Delay-dependent conditions of positivity}

Consider a scalar time-varying linear system with timevarying delays [1]:

$$
\begin{gathered}
\dot{x}(t)=a_{0}(t) x[g(t)]-a_{1}(t) x[h(t)]+b(t), \\
x(\theta)=0 \text { for } \theta<0, x(0) \in \mathbb{R},
\end{gathered}
$$

where $a_{0} \in \mathcal{L}_{\infty}, a_{1} \in \mathcal{L}_{\infty}, b \in \mathcal{L}_{\infty}, h(t)-t \in \mathcal{L}_{\infty}$, $g(t)-t \in \mathcal{L}_{\infty}$ and $h(t) \leq t, g(t) \leq t$ for all $t \geq 0$. For the system (2) the initial condition in (3) is, in general, not a continuous function (if $x(0) \neq 0$ ).

The following result proposes delay-independent positivity conditions.

Lemma 2 [1] (Corollary 15.7) Let $h(t) \leq g(t)$ and $0 \leq$ $a_{1}(t) \leq a_{0}(t)$ for all $t \geq 0$. If $x(0) \geq 0$ and $b(t) \geq 0$ for all $t \geq 0$, then the corresponding solution of (2),(3) $x(t) \geq 0$ for all $t \geq 0$.

Recall that in this case positivity is guaranteed for "discontinuous" initial conditions. The peculiarity of the condition $0 \leq a_{1}(t) \leq a_{0}(t)$ is that it may correspond to an unstable system (2). In order to overcome this issue, delay-dependent conditions can be introduced.

Lemma 3 [1] (Corollary 15.9) Let $h(t) \leq g(t)$ and $0 \leq$ $\frac{1}{e} a_{0}(t) \leq a_{1}(t)$ for all $t \geq 0$ with

$$
\sup _{t \in \mathbb{R}_{+}} \int_{h(t)}^{t}\left[a_{1}(\xi)-\frac{1}{e} a_{0}(\xi)\right] d \xi<\frac{1}{e},
$$

where $e=\exp (1)$. If $x(0) \geq 0$ and $b(t) \geq 0$ for all $t \geq 0$, then $x(t) \geq 0$ for all $t \geq 0$ in (2), (3).

These lemmas describe positivity conditions for the system (2), (3), which is more complex than (1), but scalar, they can also be extended to the $n$-dimensional system (1).

Corollary 4 The system (1) with $b(t) \geq 0$ for all $t \geq 0$ and initial conditions

$$
x(\theta)=0 \text { for }-\bar{\tau} \leq \theta<0, x(0) \in \mathbb{R}_{+}^{n},
$$

is positive if $-A_{1}$ is Metzler, $A_{0} \geq 0$, and

$$
0 \leq\left(A_{0}\right)_{i, i} \leq e\left(A_{1}\right)_{i, i}<\left(A_{0}\right)_{i, i}+\bar{\tau}^{-1}
$$

for all $i=1, \ldots, n$. 


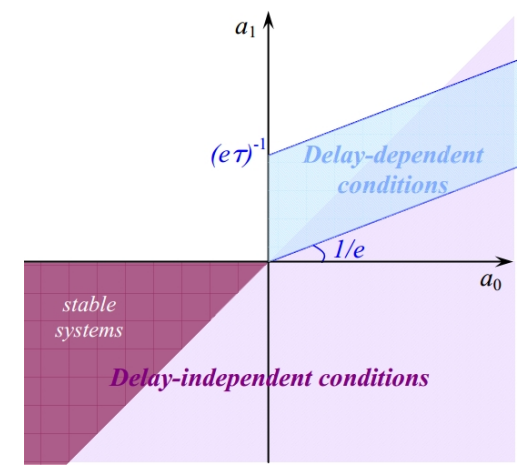

Figure 1. Different positivity conditions for (2)

From these corollaries it is easy to conclude that the delay-dependent case studied in Lemma 2 and Lemma 3 is crucially different from the delay-independent positivity conditions given first in Lemma 1, where in the scalar case the restriction $a_{1} \leq 0$ implies positivity of (1) and the condition $a_{0}<a_{1}$ according to Lemma 1 ensures stability for any $\bar{\tau}$. These results do not contradict to Remark 3.1 of [18], since $x(\theta) \neq 0$ for $-\tau \leq \theta<0$ there. A graphical illustration of different delay-independent conditions (positivity from lemmas 1 and 2) and delaydependent ones (from Lemma 3, the stability conditions are also satisfied in this case) for the system (2) is given in Fig. 1 in the plane $\left(a_{0}, a_{1}\right)$. It is worth stressing that an extension of the positivity domain in Lemma 3 is also achieved due to restrictions imposed on initial conditions in $(3)$.

In order to use the results of Lemma 2 and Lemma 3 it is necessary to pass from discontinuous initial conditions in (3) to continuous ones usually studied [31]. Further, in this section we will be interested in the case

$$
a_{0}(t)=a_{0}, a_{1}(t)=a_{1}, t-\bar{\tau} \leq h(t) \leq t, g(t)=t,
$$

where $\bar{\tau}>0$ is maximum delay. Now let us extend the initial condition (3) with a continuous one (note that all developments above can be easily adopted for piecewise continuous initial conditions, such a reformulation is omitted for simplicity):

$$
x(\theta)=\phi(\theta) \text { for } \theta \in[-\bar{\tau}, 0], \phi \in \mathcal{C}_{\bar{\tau}}
$$

and consider the conditions providing delay-dependent positivity for (2), (4), (5).

Remark 5 As mentioned in [21], the first delay interval $0 \leq t \leq \bar{\tau}$ is important for delay-dependent conditions giving solution bounds (and not just stability conditions).

Proposition 6 Let $0 \leq a_{0} \leq e a_{1}<a_{0}+\bar{\tau}^{-1}$. If $x(0) \geq$ $0, b(t) \geq 0$ for all $t \geq 0$ and

$$
b(t) \geq a_{1} \phi[h(t)] \quad \forall t \in\{0 \leq t \leq \bar{\tau}: h(t)<0\},
$$

then the corresponding solution of (2), (4), (5) satisfies $x(t) \geq 0$ for all $t \geq 0$.

PROOF. Consider the following system

$$
\begin{gathered}
\dot{z}(t)=a_{0} z(t)-a_{1} z[h(t)]+b(t)+\varphi(t), \\
z(\theta)=0 \text { for } \theta \in[-\bar{\tau}, 0), z(0)=\phi(0), \\
\varphi(t)= \begin{cases}-a_{1} \phi[h(t)] & \text { if } h(t)<0, \\
0 & \text { otherwise, }\end{cases}
\end{gathered}
$$

which is of the form (2), (4), (3) with $z(0)=\phi(0)$ and $b(t)+\varphi(t) \geq 0$ for all $t \geq 0$ by the conditions. Since all conditions of Lemma 3 are satisfied, then $z(t) \geq 0$ for all $t \geq 0$. From another side, it is easy to check that the solution of (2), (4), (5) $x(t)=z(t)$ for all $t \geq 0$.

The following extension for $n$-dimensional system (1) can be obtained.

Corollary 7 The system (1) with $b(t) \geq 0$ for all $t \geq 0$, $x(0) \in \mathbb{R}_{+}^{n}$, with a Metzler matrix $-A_{1}, A_{0} \geq 0$ and $0 \leq$ $\left(A_{0}\right)_{i, i} \leq e\left(A_{1}\right)_{i, i}<\left(A_{0}\right)_{i, i}+\bar{\tau}^{-1}$ for all $i=1, \ldots, n$, has the corresponding solution $x(t) \geq 0$ for all $t \geq 0$ provided that

$$
b(t) \geq A_{1} \phi(t-\tau(t)) \quad \forall t \in[0, \bar{\tau}] .
$$

Let us show how these conditions can be used for the design of interval observers.

\section{Interval observer design under delayed mea- surements}

In this section a useful inequality for interval analysis and a statement of the problem are given. Next, a motivating benchmark example from [23] is investigated, using the results of the previous section, in order to clarify the main idea. Finally, a delay-dependent approach for an interval observer design is presented.

\subsection{Interval bounds}

Given a matrix $A \in \mathbb{R}^{m \times n}$ define $A^{+}=\max \{0, A\}$, $A^{-}=A^{+}-A$ and $|A|=A^{+}+A^{-}$. Let $x \in \mathbb{R}^{n}$ be a vector variable, $\underline{x} \leq x \leq \bar{x}$ for some $\underline{x}, \bar{x} \in \mathbb{R}^{n}$, and $A \in \mathbb{R}^{m \times n}$ be a constant matrix, then [10]

$$
A^{+} \underline{x}-A^{-} \bar{x} \leq A x \leq A^{+} \bar{x}-A^{-} \underline{x} .
$$




\subsection{Problem statement}

Consider a linear system with a time-varying delay:

$\dot{x}(t)=A_{0} x(t)+A_{1} x[h(t)]+b(t)$,

$y(t)=C x[h(t)]+v(t)$

where $x(t) \in \mathbb{R}^{n}, t-\bar{\tau} \leq h(t) \leq t$ is a known timevarying delay $\left(t-h(t) \in \overline{\mathcal{L}}_{\infty}\right), \bar{\tau}>0$ is maximum delay, $x_{0} \in \mathcal{C}_{\bar{\tau}}^{n} ; y(t) \in \mathbb{R}^{p}$ is the system output available for measurements with the noise $v \in \mathcal{L}_{\infty}^{p} ; b \in \mathcal{L}_{\infty}^{n}$ is the system input; the constant matrices $A_{0}, A_{1}$ and $C$ have appropriate dimensions. It is assumed that for given $b$ and $h$ the system has a unique solution defined at least locally. In the state and the output equations of (7) the same delay is used, that corresponds to a delay-free system with delayed measurements, for example, or a system closed by an output-based feedback. The input $b(t)$ can be a function of control, and it can also contain a delay.

Remark 8 Note that the results to be obtained for (7) can be easily extended to the case with multiple delays:

$\dot{x}(t)=A_{0} x(t)+\sum_{i=1}^{q} A_{i} x\left[h_{i}(t)\right]+b(t)$,

$y(t)=C x\left[h_{1}(t)\right]+v(t)$,

provided that $h_{i}(t)=t-\tau_{i}(t)$ and $h_{i}(t) \leq h_{1}(t)$ for all $t \geq 0$ (in this case an output injection $y\left[t-\bar{\tau}_{i}(t)+\tau_{1}(t)\right]=$ $C x\left[h_{i}(t)\right]+v\left[t-\tau_{i}(t)+\tau_{1}(t)\right]$ can be used in observer $)$. A compact form (7) is used in the paper for brevity of presentation.

Assumption 1 There exist known functions $\underline{x}_{0}, \bar{x}_{0} \in$ $\mathcal{C}_{\bar{\tau}}^{n}$ such that $\underline{x}_{0}(\theta) \leq x_{0}(\theta) \leq \bar{x}_{0}(\theta)$ for all $\theta \in[-\bar{\tau}, 0]$.

The assumption about a known set $\left[\underline{x}_{0}, \bar{x}_{0}\right]$ for the initial conditions $x_{0}$ is standard for the interval or setmembership estimation theory $[12,17,19,20,24]$. We will assume that the values of matrices $A_{0}, A_{1}$ and $C$ are known and the instant values of the signals $b(t)$ and $v(t)$ are unavailable.

Assumption 2 There exist known signals $\underline{b}, \bar{b} \in \mathcal{L}_{\infty}^{n}$ and $\underline{v}, \bar{v} \in \mathcal{L}_{\infty}^{p}$ such that $\underline{b}(t) \leq b(t) \leq \bar{b}(t)$ and $\underline{v}(t) \leq$ $v(t) \leq \bar{v}(t)$ for all $t \geq 0$.

Therefore, the uncertain inputs $b(t), h(t)$ and $v(t)$ in (7) belong to known intervals $[\underline{b}(t), \bar{b}(t)],[t-\bar{\tau}, t]$ and $[\underline{v}(t), \bar{v}(t)]$ respectively for all $t \geq 0$.

It is required to design an interval observer,

$$
\begin{gathered}
\dot{\xi}(t)=F\left[\xi_{t}, \underline{b}(t), \bar{b}(t), \underline{v}(t), \bar{v}(t), y(t)\right], \xi_{t} \in \mathcal{C}_{\bar{\tau}}^{s}, \\
\underline{x}(t)=\underline{G}\left[\xi_{t}, \underline{b}(t), \bar{b}(t), \underline{v}(t), \bar{v}(t), y(t)\right], \\
\bar{x}(t)=\bar{G}\left[\xi_{t}, \underline{b}(t), \bar{b}(t), \underline{v}(t), \bar{v}(t), y(t)\right],
\end{gathered}
$$

such that $\underline{x}(t) \leq x(t) \leq \bar{x}(t)$ for all $t>0$ provided that $\underline{x}_{0} \leq x_{0} \leq \bar{x}_{0}$, and $x-\underline{x}, \bar{x}-x \in \mathcal{L}_{\infty}^{n}, s>0$. A similar problem has been studied in [23] but for constant delays.

\subsection{Motivating example}

Consider a motivating example introduced in [23], where the problem of a 1 -framer ${ }^{1}$ design has been posed for a scalar system

$$
\dot{x}(t)=-x(t-\tau)
$$

with initial condition $x_{0} \in \mathcal{C}_{\tau}$. This system is globally asymptotically stable if $\tau<\frac{\pi}{2}$. It has been proven in [23] (Proposition 3.2) that this system has no 1-framer of the form

$$
\begin{gathered}
F\left(\xi_{t}\right)=F_{1} \xi(t)+F_{2} \xi(t-\tau), \\
\underline{G}\left(\xi_{t}\right)=H_{1} \xi(t), \bar{G}\left(\xi_{t}\right)=H_{2} \xi(t),
\end{gathered}
$$

where $\xi_{t} \in \mathcal{C}_{\bar{\tau}}^{s}$ for any $s \geq 1$ and $F_{i}, H_{i}(i=1,2)$ are matrices of appropriate dimensions.

Applying the result of Proposition 6, the system (8) has positive solutions for a discontinuous initial condition (3) with $x(0) \geq 0$ if $\tau<\frac{1}{e}$. Actually in this case it has a non-oscillating solution which is asymptotically converging to zero ( since $\frac{1}{e}<\frac{\pi}{2}$ ), and which does not cross the zero level for all $t \in \mathbb{R}_{+}$. Further, using the result of Proposition 6, we can design a 1 -framer for (8) having the form (9) for $t \geq \tau$.

Claim 9 For the system (8) with any initial condition $x_{0} \in \mathcal{C}_{\tau}$ and $\tau<\frac{1}{e}$, the system

$$
\begin{aligned}
& \underline{\dot{x}}(t)=-\underline{x}(t-\tau)-\delta\left(\left\|\bar{x}_{0}-\underline{x}_{0}\right\|\right), \\
& \dot{\bar{x}}(t)=-\bar{x}(t-\tau)+\delta\left(\left\|\bar{x}_{0}-\underline{x}_{0}\right\|\right), \\
& \delta(s)= \begin{cases}s & \text { if } t \leq \tau, \\
0 & \text { otherwise }\end{cases}
\end{aligned}
$$

is a 1-framer, i.e. $\underline{x}(t) \leq x(t) \leq \bar{x}(t)$ for all $t>0$, provided that $\underline{x}_{0} \leq \bar{x}_{0} \leq \bar{x}_{0}, \underline{x}_{0}, \bar{x}_{0} \in \mathcal{C}_{\tau}$, and $\underline{x}, \bar{x} \in \mathcal{L}_{\infty}^{n}$.

PROOF. Introducing the interval estimation errors $\underline{e}=x-\underline{x}$ and $\bar{e}=\bar{x}-x$ we obtain

$$
\begin{gathered}
\underline{\dot{e}}=-\underline{e}(t-\tau)+\delta\left(\left\|\bar{x}_{0}-\underline{x}_{0}\right\|\right), \\
\dot{\bar{e}}=-\bar{e}(t-\tau)+\delta\left(\left\|\bar{x}_{0}-\bar{x}_{0}\right\|\right)
\end{gathered}
$$

with $\underline{e}_{0} \geq 0$ and $\bar{e}_{0} \geq 0$. All conditions of Proposition 6 are satisfied for the equations describing the error dynamics with $\tau<\frac{1}{e}$, then $\underline{e}(t) \geq 0$ and $\bar{e}(t) \geq 0$ for all $t \geq 0$.

\footnotetext{
1 The definition of a 1-framer can be found in [23], roughly speaking it is an interval open-loop estimator independent of $y(t)$.
} 
Therefore, a 1-framer of a form similar to (9) can be designed for (8) with a restricted value of delay $\tau<\frac{1}{e}$ (it differs from (9) only on the interval $[0, \tau])$. The results of simulation for this example are given in Section 5 .

Let us extend this idea of interval observer design to a more generic system (7).

\subsection{Delay-dependent conditions for interval estimation}

The equation (7) can be rewritten as follows:

$\dot{x}(t)=A_{0} x(t)+\left(A_{1}-L C\right) x[h(t)]+L y(t)+b(t)-L v(t)$,

where $L \in \mathbb{R}^{n \times p}$ is an observer gain to be designed.

Assumption 3 There exist an invertible matrix $S \in$ $\mathbb{R}^{n \times n}$ and $L \in \mathbb{R}^{n \times p}$ such that $S\left(A_{1}-L C\right) S^{-1}=R_{1}$, where $R_{1}$ is a Metzler matrix and

$$
R_{1}=R_{1}^{\dagger}+R_{1}^{2}, R_{1}^{\dagger}=\operatorname{diag}\left[-r_{1,1}, \ldots,-r_{1, n}\right], R_{1}^{2} \geq 0
$$

with $R_{1}^{\dagger}$ is the diagonal matrix composed by all elements on the main diagonal of $R_{1}, r_{1, i}>0$ for all $i=1, \ldots, n$, and $R_{1}^{2}$ is formed by the rest elements of $R_{1}$ out of the main diagonal.

The conditions of existence of such matrices $S$ and $L$ can be found in [27], in particular Assumption 3 is satisfied if the pair $\left(A_{1}, C\right)$ is observable, then they can be expressed as a LMI with respect to $S \in \mathbb{R}^{n \times n}$ and $W \in \mathbb{R}^{n \times p}$ for a fixed Metzler matrix $R_{1}$ :

$$
S A_{1}-W C=R_{1} S
$$

with $L=S^{-1} W$ (if the matrix $R_{1}$ is considered as a variable, then it is a bilinear matrix inequality; for its solution a grid of admissible values of $R_{1}$ can be used with posterior resolution of the LMI (10) for each candidate of $R_{1}$ ). Under this assumption in the new coordinates $z=S x$ the system (7) takes the form:

$$
\dot{z}(t)=R_{0} z(t)+R_{1} z[h(t)]+S L y(t)+\beta(t),
$$

where $R_{0}=S A_{0} S^{-1}$ and $\beta(t)=S[b(t)-L v(t)]$ is a new additive uncertain input, the initial condition $z_{0}=$ $S x_{0} \in \mathcal{C}_{\bar{\tau}}^{n}$ and

$$
\underline{z}_{0} \leq z_{0} \leq \bar{z}_{0}
$$

where $\underline{z}_{0}=S^{+} \underline{x}_{0}-S^{-} \bar{x}_{0}$ and $\bar{z}_{0}=S^{+} \bar{x}_{0}-S^{-} \underline{x}_{0}$ are calculated using $(6), \underline{z}_{0}, \bar{z}_{0} \in \mathcal{C}_{\bar{\tau}}^{n}$. From Assumption 2 and the relations (6) we obtain that

$$
\underline{\beta}(t) \leq \beta(t) \leq \bar{\beta}(t) \quad \forall t \geq 0,
$$

where $\underline{\beta}(t)=S^{+} \underline{b}(t)-S^{-\bar{b}}(t)-(S L)^{+} \bar{v}(t)+(S L)^{-} \underline{v}(t)$ and $\bar{\beta}(\bar{t})=S^{+} \bar{b}(t)-S^{-} \underline{b}(t)-(S L)^{+} \underline{v}(t)+(S L)^{-} \bar{v}(t)$.
Then the following interval observer can be proposed for the representation (11):

$$
\begin{aligned}
\underline{\dot{z}}(t) & =R_{0}^{+} \underline{z}(t)-R_{0}^{-} \bar{z}(t)+R_{1} \underline{z}[h(t)]+S L y(t)+\underline{\beta}(t)-\delta, \\
\dot{\bar{z}}(t) & =R_{0}^{+} \bar{z}(t)-R_{0}^{-} \underline{z}(t)+R_{1} \bar{z}[h(t)]+S L y(t)+\bar{\beta}(t)+\delta, \\
\delta & =\left[\delta_{1}, \ldots, \delta_{n}\right]^{\mathrm{T}}, \\
\delta_{i} & =\left\{\begin{array}{ll}
r_{1, i}|| \bar{z}_{0, i}-\underline{z}_{0, i} \| & \text { if } t \leq \bar{\tau} \\
0 & \text { otherwise }
\end{array}, i=1, \ldots n\right.
\end{aligned}
$$

with initial conditions $\underline{z}_{0}, \bar{z}_{0}$ for the variables $\underline{z}(t), \bar{z}(t)$ respectively. Finally interval estimates for the variable $x(t)$ can also be obtained using (6):

$\underline{x}(t)=\left(S^{-1}\right)^{+} \underline{z}(t)-\left(S^{-1}\right)^{-} \bar{z}(t)$,

$\bar{x}(t)=\left(S^{-1}\right)^{+} \bar{z}(t)-\left(S^{-1}\right)^{-} \underline{z}(t)$,

which may be conservative, see discussion and improved solutions in [29]. For all $i=1, \ldots, n$ denote

$$
r_{0, i}=\left(R_{0}^{+}\right)_{i, i} .
$$

Proposition 10 Let assumptions 1-3 be satisfied and

$$
r_{0, i} \leq e r_{1, i}<r_{0, i}+\bar{\tau}^{-1}
$$

for all $i=1, \ldots, n$. Then the relations

$$
\underline{x}(t) \leq x(t) \leq \bar{x}(t) \quad \forall t \geq 0
$$

hold for the system (7) and the interval observer (12), (13). If in addition there exist symmetric matrices $P \in$ $\mathbb{R}^{2 n \times 2 n}, \Sigma \in \mathbb{R}^{2 n \times 2 n}, \Xi \in \mathbb{R}^{2 n \times 2 n}$ and $\Theta \in \mathbb{R}^{2 n \times 2 n}$ such that the LMIs

$$
\begin{gathered}
{\left[\begin{array}{ll}
\Xi & \Theta \\
\Theta & \Xi
\end{array}\right] \succeq 0, P \succ 0, \Sigma \succ 0, \Xi \succ 0,} \\
{\left[\begin{array}{cccc}
\Phi_{0}^{T} P+P \Phi_{0}+\Sigma-\Xi & \Theta & P \Phi_{1}+\Xi-\Theta & \bar{\tau} \Phi_{0}^{T} \Xi \\
\Theta & -\Sigma-\Xi & \Xi-\Theta & 0_{2 n \times 2 n} \\
\Phi_{1}^{T} P+\Xi-\Theta & \Xi-\Theta & \Theta+\Theta^{T}-2 \Xi & \bar{\tau} \Phi_{1}^{T} \Xi \\
\bar{\tau} \Xi \Phi_{0} & 0_{2 n \times 2 n} & \bar{\tau} \Xi \Phi_{1} & -\Xi
\end{array}\right] \prec 0,}
\end{gathered}
$$

where

$$
\Phi_{0}=\left[\begin{array}{cc}
R_{0}^{+} & R_{0}^{-} \\
R_{0}^{-} & R_{0}^{+}
\end{array}\right], \Phi_{1}=\left[\begin{array}{cc}
R_{1} & 0_{n \times n} \\
0_{n \times n} & R_{1}
\end{array}\right]
$$

are satisfied, then $x-\underline{x}, \bar{x}-x \in \mathcal{L}_{\infty}^{n}$.

PROOF. Introduce the interval estimation errors $\underline{e}=$ $z-\underline{z}$ and $\bar{e}=\bar{z}-z$ for the observer (12) and (11):

$\underline{\dot{e}}(t)=R_{0}^{+} \underline{e}(t)+R_{0}^{-} \bar{e}(t)+R_{1} \underline{e}[h(t)]+\beta(t)-\beta(t)+\delta$,

$\dot{\bar{e}}(t)=R_{0}^{+} \bar{e}(t)+R_{0}^{-} \underline{e}(t)+R_{1} \bar{e}[h(t)]+\bar{\beta}(t)-\beta(t)+\delta$, 
which for any $i=1, \ldots, n$ may be rewritten as follows:

$$
\begin{aligned}
\underline{\dot{e}}_{i}(t)= & r_{0, i} \underline{e}_{i}(t)-r_{1, i} \underline{e}_{i}[h(t)]+\underline{\chi}_{i}(t) \\
& +\beta_{i}(t)-\underline{\beta}_{i}(t)+\delta_{i}, \\
\dot{\bar{e}}_{i}(t)= & r_{0, i} \bar{e}_{i}(t)-r_{1,0} \bar{e}_{i}[h(t)]+\bar{\chi}_{i}(t) \\
& +\bar{\beta}_{i}(t)-\beta_{i}(t)+\delta_{i},
\end{aligned}
$$

where

$$
\begin{aligned}
& \underline{\chi}_{i}(t)=\sum_{j=1, j \neq i}^{n}\left(R_{0}^{+}\right)_{i, j} \underline{e}_{j}(t)+\sum_{k=1}^{n}\left(R_{0}^{-}\right)_{i, k} \bar{e}_{k}(t)+\sum_{j=1}^{n}\left(R_{1}^{2}\right)_{i, j} \underline{e}_{j}(t), \\
& \bar{\chi}_{i}(t)=\sum_{j=1, j \neq i}^{n}\left(R_{0}^{+}\right)_{i, j} \bar{e}_{j}(t)+\sum_{k=1}^{n}\left(R_{0}^{-}\right)_{i, k} \underline{e}_{k}(t)+\sum_{j=1}^{n}\left(R_{1}^{2}\right)_{i, j} \bar{e}_{j}(t) .
\end{aligned}
$$

The relations $\beta_{i}(t)-\underline{\beta}_{i}(t) \geq 0, \bar{\beta}_{i}(t)-\beta_{i}(t) \geq 0$ for all $t \geq 0$ and $\delta \geq 0$ are satisfied by construction, for all $i=1, \ldots, n$. The signals $\underline{\chi}_{i}(t) \geq 0$ and $\bar{\chi}_{i}(t) \geq 0$ for all $t \geq 0$ and $i=1, \ldots, n$ provided that $\underline{e}(t) \geq 0$ and $\bar{e}(t) \geq$ 0 . Note that for the systems (16), (17) all conditions of Proposition 6 are satisfied due to the selection of $\delta$, thus by induction if $\underline{e}(0) \geq 0$ and $\bar{e}(0) \geq 0$, this property is preserved for all $t \geq 0$ :

$$
\underline{e}(t) \geq 0, \bar{e}(t) \geq 0
$$

Therefore, from (13) the required property (14) is valid.

In order to prove boundedness of $x-\underline{x}, \bar{x}-x$ consider a Lyapunov functional candidate from $[25,16]$ :

$$
\begin{aligned}
V\left(t, \zeta_{t}, \dot{\zeta}_{t}\right)= & \zeta^{T}(t) P \zeta(t)+\int_{t-\bar{\tau}}^{t} \zeta^{T}(s) \Sigma \zeta(s) d s \\
& +\bar{\tau} \int_{-\bar{\tau}}^{0} \int_{t+\theta}^{t} \dot{\zeta}^{T}(s) \Xi \dot{\zeta}(s) d s d \theta,
\end{aligned}
$$

where $\zeta=\left[\underline{e}^{T} \bar{e}^{T}\right]^{T}$ is the combined error vector of the observer (12), and dynamics of $\zeta$ have the form:

$\dot{\zeta}(t)=\Phi_{0} \zeta(t)+\Phi_{1} \zeta[h(t)]+\left[\begin{array}{l}\beta(t)-\underline{\beta}(t) \\ \bar{\beta}(t)-\beta(t)\end{array}\right]+\left[\begin{array}{c}I_{n} \\ I_{n}\end{array}\right] \delta$

where the matrices $\Phi_{0}$ and $\Phi_{1}$ are defined in the proposition formulation. The LMIs (15) imply stability of this system $[25,16]$, and boundedness of $\zeta(t)$ for any bounded input.

The LMIs (15) ensure stability of (16), (17), they can be modified $[16]$ in order to ensure a desired gain from the inputs $\beta-\beta, \bar{\beta}-\beta$ to the estimation errors $\underline{e}, \bar{e}$ optimizing the interval estimation accuracy. Such a modification is omitted for brevity of presentation.
Remark 11 The restrictions imposed in Proposition 10 on all matrices $S, L, P, \Sigma, \Xi$ and $\Theta$, which are needed to design interval observer (12), are interrelated and nonlinear, therefore, it is hard to represent them in a LMI form directly. It is proposed to decouple these conditions: on the LMI (10) from Assumption 3 (if $L$ and $S$ are fixed, then the matrices $R_{0}$ and $R_{1}$ become given, and vice versa) and the $L M I$ (15) with respect to $P, \Sigma, \Xi$ and $\Theta$. Such a two step scheme can be iterated for different selections of $R_{1}$, until a solution is found.

An alternative procedure can be provided assuming that $S=I_{n}$ and $\Xi=\mu P$ for some scalar tuning parameter $\mu>0$, then the above conditions can be rewritten as a series of bilinear matrix inequalities:

$$
\begin{aligned}
& P \Phi_{1}-W\left[\begin{array}{l}
C \\
C
\end{array}\right]+\Upsilon \geq 0, P>0, \Sigma \succ 0, \Upsilon \geq 0, \\
& {\left[\begin{array}{cc}
\mu P & \Theta \\
\Theta & \mu P
\end{array}\right] \succeq 0, \Phi_{0}=\left[\begin{array}{cc}
A_{0}^{+} & A_{0}^{-} \\
A_{0}^{-} & A_{0}^{+}
\end{array}\right], \Phi_{1}=\left[\begin{array}{cc}
A_{1} & 0_{n \times n} \\
0_{n \times n} & A_{1}
\end{array}\right],} \\
& {\left[\begin{array}{cccc}
\Phi_{0}^{T} P+P \Phi_{0}+\Sigma-\mu P & \Theta & \Phi_{2} & \bar{\tau} \mu \Phi_{0}^{T} P
\end{array}\right]} \\
& \left.\begin{array}{cccc}
\Theta & -\Sigma-\mu P & \mu P-\Theta & 0_{2 n \times 2 n} \\
\Phi_{2}^{T} & \mu P-\Theta & \Theta+\Theta^{T}-2 \mu P & \Phi_{3}^{T} \\
\bar{\tau} \mu P \Phi_{0} & 0_{2 n \times 2 n} & \Phi_{3} & -\mu P
\end{array}\right] \prec 0, \\
& \Phi_{2}=P \Phi_{1}-W\left[\begin{array}{l}
C \\
C
\end{array}\right]+\mu P-\Theta, \Phi_{3}=\bar{\tau} \mu\left(P \Phi_{1}-W\left[\begin{array}{l}
C \\
C
\end{array}\right]\right),
\end{aligned}
$$

for symmetric matrices $P \in \mathbb{R}^{2 n \times 2 n}, \Sigma \in \mathbb{R}^{2 n \times 2 n}, \Upsilon \in$ $\mathbb{R}^{2 n \times 2 n}$ and $\Theta \in \mathbb{R}^{2 n \times 2 n}, \Upsilon$ and $P$ should also be declared diagonal, $W \in \mathbb{R}^{2 n \times 2 p}$ is a matrix block-diagonal variable, then $L=P^{-1} W$. For any fixed value of $\mu$ the above system becomes a LMI and it can be efficiently solved with respect to $L, P, \Sigma$ and $\Theta$ (and $W, \Upsilon)$.

The result of Proposition 10 is based on a rather restrictive Assumption 3, that the matrix $A_{1}-L C$ is Hurwitz. In many cases (if, for example, the output $y$ measurements are available with delays, but the system itself has no delayed dynamics) this assumption cannot be verified and may be relaxed as follows.

Assumption 4 There exist an invertible matrix $S \in$ $\mathbb{R}^{n \times n}$ and $L \in \mathbb{R}^{n \times p}$ such that

$$
\begin{gathered}
S\left(A_{1}-L C\right) S^{-1}=Q_{1}=\left[\begin{array}{cc}
\bar{Q}_{1} & 0_{l \times n-l} \\
0_{n-l \times l} & 0_{n-l \times n-l}
\end{array}\right], \\
S A_{0} S^{-1}=Q_{0}=\left[\begin{array}{cc}
Q_{0,1} & Q_{0,2} \\
Q_{0,3} & Q_{0,4}
\end{array}\right], \\
Q_{0,1} \in \mathbb{R}^{l \times l}, Q_{0,2} \in \mathbb{R}^{l \times n-l}, Q_{0,3} \in \mathbb{R}^{n-l \times l}, \\
Q_{0,4} \in \mathbb{R}^{n-l \times n-l}, \bar{Q}_{1}=\bar{Q}_{1}^{\dagger}+\bar{Q}_{1}^{2}, Q_{0,4}=Q_{0,4}^{\dagger}+Q_{0,4}^{2}, \\
\text { where } \bar{Q}_{1}^{\dagger}=\operatorname{diag}^{\dagger}\left[-q_{1,1}, \ldots,-q_{1, l}\right] \text { with } q_{1, k}>0 \text { for all } \\
k=1, \ldots, l, \bar{Q}_{1}^{2} \geq 0, \text { and } 0<l \leq n .
\end{gathered}
$$


As before $\bar{Q}_{1}^{\dagger}, Q_{0,4}^{\dagger}$ are diagonal matrices composed by all elements on the main diagonals of $\bar{Q}_{1}$ and $Q_{0,4}$ respectively, and $\bar{Q}_{1}^{l}, Q_{0,4}^{2}$ are formed by the rest elements of $\bar{Q}_{1}$ and $Q_{0,4}$ out of the main diagonals. In this case it is assumed that some part of the system (7) cannot be stabilized by a linear output injection. In the new coordinates $z=S x=\left[\begin{array}{ll}z_{1}^{\mathrm{T}} & z_{2}^{\mathrm{T}}\end{array}\right]^{\mathrm{T}}, z_{1} \in \mathbb{R}^{l}, z_{2} \in \mathbb{R}^{n-l}$ the system (7) takes the form:

$$
\begin{aligned}
& \dot{z}_{1}(t)=\bar{Q}_{0} z(t)+\bar{Q}_{1} z_{1}[h(t)]+\Lambda_{1} y(t)+\beta_{1}(t), \\
& \dot{z}_{2}(t)=Q_{0,3} z_{1}(t)+Q_{0,4} z_{2}(t)+\Lambda_{2} y(t)+\beta_{2}(t),
\end{aligned}
$$

where $\bar{Q}_{0}=\left[Q_{0,1} Q_{0,2}\right]$ and $S L=\left[\Lambda_{1}^{\mathrm{T}} \Lambda_{2}^{\mathrm{T}}\right]^{\mathrm{T}}$ are the matrices of appropriate dimensions; and the input $\beta(t)=$ $\left[\beta_{1}^{\mathrm{T}}(t) \beta_{2}^{\mathrm{T}}(t)\right]^{\mathrm{T}}=S[b(t)-L v(t)]$ with the initial condition $z_{0}=\left[\begin{array}{ll}z_{01}^{\mathrm{T}} & z_{02}^{\mathrm{T}}\end{array}\right]^{\mathrm{T}}=S x_{0} \in \mathcal{C}_{\bar{\tau}}^{n}$ have the same form and interval bounds as for (11). Then the following interval observer can be proposed for the representation (19) instead of (12):

$$
\begin{aligned}
\underline{\dot{z}}_{1}(t)= & \bar{Q}_{0}^{+} \underline{z}(t)-\bar{Q}_{0}^{-} \bar{z}(t)+\bar{Q}_{1} \underline{z}_{1}[h(t)]+\Lambda_{1} y(t)+\underline{\beta}_{1}(t)-\delta^{l}, \\
\dot{\bar{z}}_{1}(t)= & \bar{Q}_{0}^{+} \bar{z}(t)-\bar{Q}_{0}^{-} \underline{z}(t)+\bar{Q}_{1} \bar{z}_{1}[h(t)]+\Lambda_{1} y(t)+\bar{\beta}_{1}(t)+\delta^{l}, \\
\dot{z}_{2}(t)= & Q_{0,3}^{+} \underline{z}_{1}(t)-Q_{0,3}^{-} \bar{z}_{1}(t)+Q_{0,4}^{\dagger} \underline{z}_{2}(t)+\left(Q_{0,4}^{2}\right)^{+} \underline{z}_{2}(t) \\
& -\left(Q_{0,4}^{2}\right)^{-} \bar{z}_{2}(t)+\Lambda_{2} y(t)+\underline{\beta}_{2}(t), \\
\dot{\bar{z}}_{2}(t)= & Q_{0,3}^{+} \bar{z}_{1}(t)-Q_{0,3}^{-} \underline{z}_{1}(t)+Q_{0,4}^{\dagger} \bar{z}_{2}(t)+\left(Q_{0,4}^{2}\right)^{+} \bar{z}_{2}(t) \\
& -\left(Q_{0,4}^{2}\right)^{-} \underline{z}_{2}(t)+\Lambda_{2} y(t)+\bar{\beta}_{2}(t), \\
\delta^{l}= & {\left[\delta_{1}^{l}, \ldots, \delta_{l}^{l}\right]^{\mathrm{T}}, } \\
\delta_{k}^{l}= & \left\{\begin{array}{ll}
q_{1, k}\left\|\bar{z}_{0, k}-\underline{z}_{0, k}\right\| & \text { if } t \leq \bar{\tau} \\
0 & \text { otherwise }
\end{array}, k=1, \ldots l\right.
\end{aligned}
$$

with initial conditions $\underline{z}_{0}, \bar{z}_{0} \in \mathcal{C}_{\bar{\tau}}^{n}$ for the variables $\underline{z}(t)=\left[\underline{z}_{1}^{\mathrm{T}}(t) \underline{z}_{2}^{\mathrm{T}}(t)\right]^{\mathrm{T}}, \bar{z}(t)=\left[\bar{z}_{1}^{\mathrm{T}}(t) \bar{z}_{2}^{\mathrm{T}}(t)\right]^{\mathrm{T}}$ respectively. Finally interval estimates for the variable $x(t)$ can also be obtained using (13). For all $k=1, \ldots, l$ denote

$$
q_{0, k}=\left(\bar{Q}_{0}^{+}\right)_{k, k}
$$

Theorem 12 Let assumptions 1, 2 and 4 be satisfied and

$$
q_{0, k} \leq e q_{1, k}<q_{0, k}+\bar{\tau}^{-1}
$$

for all $k=1, \ldots, l$. Then the interval observer (13), (20) for the system (7) admits the relations (14). If in addition there exist symmetric matrices $P \in \mathbb{R}^{2 n \times 2 n}$, $\Sigma \in \mathbb{R}^{2 n \times 2 n}, \Xi \in \mathbb{R}^{2 n \times 2 n}$ and $\Theta \in \mathbb{R}^{2 n \times 2 n}$ such that the LMIs (15) are satisfied for

$$
\begin{gathered}
\Phi_{0}=\left[\begin{array}{cccc}
Q_{01}^{+} & Q_{01}^{-} & Q_{02}^{+} & Q_{02}^{-} \\
Q_{01}^{-} & Q_{01}^{+} & Q_{02}^{-} & Q_{02}^{+} \\
Q_{0,3}^{+} & Q_{0,3}^{-} & Q_{0,4}^{\dagger}+\left(Q_{0,4}^{2}\right)^{+} & \left(Q_{0,4}^{2}\right)^{-} \\
Q_{0,3}^{-} & Q_{0,3}^{+} & \left(Q_{0,4}^{2}\right)^{-} & Q_{0,4}^{\dagger}+\left(Q_{0,4}^{2}\right)^{+}
\end{array}\right], \\
\Phi_{1}=\left[\begin{array}{cccc}
\bar{Q}_{1} & 0_{l \times l} & 0_{l \times n-l} & 0_{l \times n-l} \\
0_{l \times l} & \bar{Q}_{1} & 0_{l \times n-l} & 0_{l \times n-l} \\
0_{n-l \times l} & 0_{n-l \times l} & 0_{n-l \times n-l} & 0_{n-l \times n-l} \\
0_{n-l \times l} & 0_{n-l \times l} & 0_{n-l \times n-l} & 0_{n-l \times n-l}
\end{array}\right],
\end{gathered}
$$

then $x-\underline{x}, \bar{x}-x \in \mathcal{L}_{\infty}^{n}$.

PROOF. Introduce the interval estimation errors $\underline{e}=$ $z-\underline{z}=\left[\underline{e}_{1}^{\mathrm{T}} \underline{e}_{2}^{\mathrm{T}}\right]^{\mathrm{T}}$ and $\bar{e}=\bar{z}-z=\left[\begin{array}{ll}\bar{e}_{1}^{\mathrm{T}} & \bar{e}_{2}^{\mathrm{T}}\end{array}\right]^{\mathrm{T}}$ for the observer (20) and (19):

$$
\begin{aligned}
\dot{e}_{1}(t)= & \bar{Q}_{0}^{+} \underline{e}(t)+\bar{Q}_{0}^{-} \bar{e}(t)+\bar{Q}_{1} \underline{e}_{1}[h(t)]+\beta_{1}(t)-\underline{\beta}_{1}(t)+\delta^{l}, \\
\dot{\bar{e}}_{1}(t)= & \bar{Q}_{0}^{+} \bar{e}(t)+\bar{Q}_{0}^{-} \underline{e}(t)+\bar{Q}_{1} \bar{e}_{1}[h(t)]+\bar{\beta}_{1}(t)-\beta_{1}(t)+\delta^{l}, \\
\dot{e}_{2}(t)= & Q_{0,3}^{+} \underline{e}_{1}(t)+Q_{0,3}^{-} \bar{e}_{1}(t)+\left[Q_{0,4}^{\dagger}+\left(Q_{0,4}^{2}\right)^{+}\right] \underline{e}_{2}(t) \\
& +\left(Q_{0,4}^{2}\right)^{-} \bar{e}_{2}(t)+\beta_{2}(t)-\underline{\beta}_{2}(t), \\
\dot{\bar{e}}_{2}(t)= & Q_{0,3}^{+} \bar{e}_{1}(t)+Q_{0,3}^{-} \underline{e}_{1}(t)+\left[Q_{0,4}^{\dagger}+\left(Q_{0,4}^{2}\right)^{+}\right] \bar{e}_{2}(t) \\
& +\left(Q_{0,4}^{2}\right)^{-} \underline{e}_{2}(t)+\bar{\beta}_{2}(t)-\beta_{2}(t) .
\end{aligned}
$$

It is easy to see that positivity analysis for the variables $\underline{e}_{1}(t)$ and $\bar{e}_{1}(t)$ is similar to the one given in the proof of Proposition 10, while for the variables $\underline{e}_{2}(t)$ and $\bar{e}_{2}(t)$ the positivity follows the fact that the matrix $Q_{0,4}^{\dagger}+\left(Q_{0,4}^{2}\right)^{+}$ is Metzler by construction and the rest terms in the righthand side of $\underline{\dot{e}}_{2}, \dot{\bar{e}}_{2}$ are nonnegative provided that $\underline{e}(t) \geq 0$ and $\bar{e}(t) \geq 0$. By induction, if $\underline{e}(0) \geq 0$ and $\bar{e}(\overline{0}) \geq 0$, then the relations $\underline{e}(t) \geq 0, \bar{e}(t) \geq 0$ are preserved for all $t \geq 0$ [32]. Therefore, from (13) the inclusion (14) is valid.

In order to prove boundedness of $\underline{x}, \bar{x}$ consider a Lyapunov functional candidate $(18)$ from $[25,16]$, where $\zeta=$ $\left[\begin{array}{llll}\underline{e}_{1}^{T} & \bar{e}_{1}^{T} & \underline{e}_{2}^{T} & \bar{e}_{2}^{T}\end{array}\right]^{T}$ with

$$
\dot{\zeta}(t)=\Phi_{0} \zeta(t)+\Phi_{1} \zeta[h(t)]+\left[\begin{array}{c}
\beta_{1}(t)-\underline{\beta}_{1}(t) \\
\bar{\beta}_{1}(t)-\beta_{1}(t) \\
\beta_{2}(t)-\underline{\beta}_{2}(t) \\
\bar{\beta}_{2}(t)-\beta_{2}(t)
\end{array}\right]+\left[\begin{array}{c}
I_{l} \\
I_{l} \\
0_{n-l \times l} \\
0_{n-l \times l}
\end{array}\right] \delta^{l}
$$

and the matrices $\Phi_{0}$ and $\Phi_{1}$ are defined in the theorem formulation. The LMIs (15) imply stability of this system $[25,16]$, and boundedness of solutions for any bounded inputs. 
Remark 13 It has been assumed before that the delay $h(t)$ is time-varying and known, the latter restriction can be relaxed rewriting the equations (19) as follows:

$$
\begin{aligned}
\dot{z}_{1}(t)= & \bar{Q}_{0} z(t)+\bar{Q}_{1} z_{1}[t-\bar{\tau}]+\bar{Q}_{1}\left\{z_{1}[h(t)]-z_{1}[t-\bar{\tau}]\right\} \\
& +\Lambda_{1} y(t)+\beta_{1}(t) \\
\dot{z}_{2}(t)= & Q_{0,3} z_{1}(t)+Q_{0,4} z_{2}(t)+\Lambda_{2} y(t)+\beta_{2}(t)
\end{aligned}
$$

where as in [12] it is possible to calculate an interval inclusion:

$$
\begin{gathered}
\underline{q}_{1}(t) \leq \bar{Q}_{1}\left\{z_{1}[h(t)]-z_{1}[t-\bar{\tau}]\right\} \leq \bar{q}_{1}(t), \\
\underline{q}_{1}(t)=\min _{s \in[t-\bar{\tau}, t]}\left\{\bar{Q}_{1}^{\dagger}\left(\bar{z}_{1}[s]-\underline{z}_{1}[t-\bar{\tau}]\right)+\bar{Q}_{1}^{2}\left(\underline{z}_{1}[s]-\bar{z}_{1}[t-\bar{\tau}]\right)\right\}, \\
\bar{q}_{1}(t)=\max _{s \in[t-\bar{\tau}, t]}\left\{\bar{Q}_{1}^{\dagger}\left(\underline{z}_{1}[s]-\bar{z}_{1}[t-\bar{\tau}]\right)+\bar{Q}_{1}^{2}\left(\bar{z}_{1}[s]-\underline{z}_{1}[t-\bar{\tau}]\right)\right\}
\end{gathered}
$$

provided that $\underline{z}_{1}(\theta) \leq z_{1}(\theta) \leq \bar{z}_{1}(\theta)$ for all $\theta \geq-\bar{\tau}$, where $\underline{q}_{1}(t)$ and $\bar{q}_{1}(t)$ can be derived on-line. Then the term containing uncertain time-varying delay $\bar{Q}_{1}\left\{z_{1}[h(t)]-\right.$ $\left.z_{1}[t-\bar{\tau}]\right\}$ can be treated as a part of $\beta_{1}(t)$, and interval observer (13), (20) can be applied taking into account only the maximal admissible delay $\bar{\tau}$ (see the pendulum example in Section 5). Another approach that can be used to treat uncertain time-varying delays (skipping $q_{1}(t)$ and $\left.\bar{q}_{1}(t)\right)$ is presented in [28].

Remark 14 Though all results in the paper are formulated for a linear system (7), they can also be applied to nonlinear ones, provided that nonlinearities are functions of measured outputs and inputs. Such a case is illustrated by the pendulum example below.

\section{$5 \quad$ Examples}

\subsection{Motivating example}

To illustrate the result of Claim 9 for the system (8) let us consider ( 7 ) for $n=1$

$$
\dot{x}(t)=u(t)+d(t), y(t)=x(t-\tau(t))+v(t),
$$

where $x(t) \in \mathbb{R}$ is the state, $u(t)=\sin (t)$ is the system known input, $d(t) \in[-0.1,0.1]$ is the input disturbance, $v(t) \in[-0.1,0.1]$ is the measurement noise, and $\tau(t)=$ $\frac{1}{2.02 e}(1+\sin (0.5 t))$ with $\bar{\tau}=\frac{1}{1.01 e}$. We can rewrite this system as follows:

$$
\begin{gathered}
\dot{x}(t)=-x(t-\tau(t))+b(t), \\
b(t)=y(t)+u(t)+d(t)-v(t)
\end{gathered}
$$

with $\underline{b}(t)=y(t)+u(t)-0.2$ and $\bar{b}(t)=y(t)+u(t)+0.2$, where now $x(\theta)=x(0)$ for all $\theta \in[-\bar{\tau}, 0)$. Assume that $\left\|x_{0}\right\| \leq 5$. For $L=0$ and $S=1$ the interval observer (12) takes a form similar to the 1-framer from Claim 9,

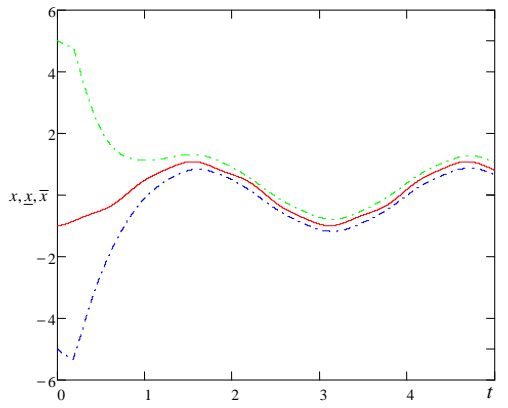

Figure 2. The results of simulation for the motivating example

and all conditions of this claim or Proposition 10 are satisfied. The results of simulation for

$$
d(t)=0.1 \cos (3 t), v(t)=0.1 \sin (5 t)
$$

are shown in Fig. 2. The red solid curve represents a trajectory of the system $x(t)$, the blue and green dash-dot lines correspond to the interval estimates $\underline{x}(t)$ and $\bar{x}(t)$ generated by the interval observer. As we can conclude from Fig. 2, the inclusion $\underline{x}(t) \leq x(t) \leq \bar{x}(t)$ is ensured for all $t \geq 0$, and asymptotically the width of the interval $[\underline{x}(t), \bar{x}(\bar{t})]$ is proportional to the system uncertainty.

\subsection{A pendulum example}

Consider an example of (7) for $n=2$

$$
\begin{aligned}
\dot{x}_{1}(t)= & x_{2}(t), y(t)=x_{1}(t-\tau(t)) \\
\dot{x}_{2}(t)= & 0.1 x_{1}(t)-0.5 x_{2}(t)-0.35 \sin \left[x_{1}(t-\hat{\tau}(t))\right] \\
& +\sin (1.25 t)
\end{aligned}
$$

where $0 \leq \tau(t) \leq \bar{\tau}$ is a delay of measurements and $1 \leq \hat{\tau}(t) \leq 2$ is an uncertain time varying delay in the state equation (for simulation we selected $\tau(t)=$ $0.24+0.12 \sin (t)$ and $\left.\hat{\tau}(t)=1+\sin ^{2}(2 t)\right)$. The models of nonlinear delayed pendulums appear in microgrid control systems [11]. Thus, in this case

$$
\begin{gathered}
A_{0}=\left[\begin{array}{cc}
0 & 1 \\
0.1 & -0.5
\end{array}\right], A_{1}=0, \\
h(t)=t-\tau(t), \underline{\tau}=0.36, v(t)=0, \\
0 \\
b(t)=\left[\begin{array}{c}
0 \\
-0.35 \sin [y(t-\hat{\tau}(t)+\tau(t))]+\sin (1.25 t)
\end{array}\right],
\end{gathered}
$$

and Assumption 2 is satisfied for:

$$
\begin{aligned}
& \underline{b}(t)=\left[\begin{array}{c}
0 \\
\min _{\theta \in[1,2]}\{-0.35 \sin [y(t-\theta+\tau(t))]\}+\sin (1.25 t)
\end{array}\right], \\
& \underline{b}(t)=\left[\begin{array}{c}
0 \\
\max _{\theta \in[1,2]}\{-0.35 \sin [y(t-\theta+\tau(t))]\}+\sin (1.25 t)
\end{array}\right] .
\end{aligned}
$$




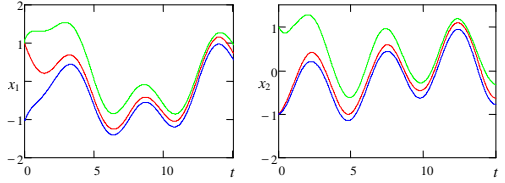

Figure 3. The results of simulation for the delayed pendulum

The results of simulation in Fig. 3 show that for the initial conditions $\left\|x_{10}\right\| \leq 1,\left\|x_{20}\right\| \leq 1$ Assumption 1 is also satisfied. For $L=\left[\begin{array}{ll}1 & 0\end{array}\right]^{T}$ and $S=I_{2}$ the conditions of Assumption 4 are verified, then $z=x$. The LMIs of Theorem 12 are satisfied for the given value of $\bar{\tau}=0.36$. The results of simulation are shown in Fig. 3, they confirm efficiency of interval estimation and validity of delay-dependent positivity conditions (the stability conditions of Theorem 12 are satisfied for $\bar{\tau} \leq 1.3$ in this example, but for $0.37 \leq \bar{\tau} \leq 1.3$ the positivity conditions of Proposition 6 are not satisfied and the interval estimation cannot be guaranteed).

\section{Conclusion}

In the paper, new interval observers for linear time-delay systems with delayed measurements have been designed extending the theory of $[23,12,14]$. For this goal, new delay-dependent positivity conditions for linear systems with time-varying delays have been proposed. These conditions are related with non-oscillatory behavior of solutions [1]. They nicely complement the existing delayindependent conditions of [18] (see Fig. 1). The results have been applied for the benchmark system from [23]. Two interval observers have been proposed for the cases of observable or detectable systems. The efficacy of observers has been illustrated by numerical experiments. Extension of these results for the case of sampled-data measurements is a direction of future research.

\section{Acknowledgements}

This work was partially supported by the Government of Russian Federation (Grant 074-U01), the Ministry of Education and Science of Russian Federation (Project 14.Z50.31.0031) and by Israel Science Foundation (grant No 1128/14).

\section{References}

[1] Ravi P. Agarwal, Leonid Berezansky, Elena Braverman, and Alexander Domoshnitsky. Nonoscillation theory of functional differential equations with applications. Springer, New York, 2012.

[2] Mustapha Ait Rami. Stability analysis and synthesis for linear positive systems with time-varying delays. In Rafael Bru and Sergio Romero-Vivó, editors, Positive Systems, volume 389 of Lecture Notes in Control and Information Sciences, pages 205-215. Springer, Berlin Heidelberg, 2009.
[3] P. Borne, M. Dambrine, W. Perruquetti, and J.-P. Richard. Stability Theory at the end of the XXth Century, chapter Vector Lyapunov Function: Nonlinear, TimeVarying, Ordinary and Functional Differential Equations, pages 49-73. Taylor \& Francis, London, 2003.

[4] C. Briat, O. Sename, and J.-F. Lafay. Design of LPV observers for LPV time-delay systems: an algebraic approach. Int. J. Control, 84(9):1533-1542, 2011.

[5] C. Califano, L.A. Marquez-Martinez, and C.H. Moog. On the observer canonical form for nonlinear time-delay systems. In Proc. 18th IFAC World Congress, Milano, 2011.

[6] M. Dambrine and J.P. Richard. Stability analysis of timedelay systems. Dynamic Systems and Applications, 2(3):405414, 1993.

[7] M. Dambrine and J.P. Richard. Stability and stability domains analysis for nonlinear differential-difference equations. Dynamic Systems and Applications, 3(3):369-378, 1994.

[8] M. Dambrine, J.P. Richard, and P. Borne. Feedback control of time-delay systems with bounded control and state. Mathematical Problems in Engineering, 1(1):77-87, 1995.

[9] A. Domoshnitsky. Maximum principles and nonoscillation intervals for first order Volterra functional differential equations. Dynamics of Continuous, Discrete 8 Impulsive Systems. A: Mathematical Analysis, 15:769-814, 2008.

[10] D. Efimov, L.M. Fridman, T. Raïssi, A. Zolghadri, and R. Seydou. Interval estimation for LPV systems applying high order sliding mode techniques. Automatica, 48:23652371, 2012.

[11] D. Efimov, R. Ortega, and J. Schiffer. ISS of multistable systems with delays: application to droop-controlled inverterbased microgrids. In Proc. ACC'15, Chicago, 2015.

[12] D. Efimov, W. Perruquetti, and J.-P. Richard. Interval estimation for uncertain systems with time-varying delays. International Journal of Control, 86(10):1777-1787, 2013.

[13] D. Efimov, A. Polyakov, E.M. Fridman, W. Perruquetti, and J.-P. Richard. Delay-dependent positivity: Application to interval observers. In Proc. ECC'15, Linz, 2015.

[14] D. Efimov, A. Polyakov, and J.-P. Richard. Interval observer design for estimation and control of time-delay descriptor systems. European Journal of Control, 23(5):26-35, 2015.

[15] Emilia Fridman. Introduction to Time-Delay Systems: Analysis and Control. Birkhuser, Basel, 2014.

[16] Emilia Fridman. Tutorial on Lyapunov-based methods for time-delay systems. European Journal of Control, 2014.

[17] J.L. Gouzé, A. Rapaport, and M.Z. Hadj-Sadok. Interval observers for uncertain biological systems. Ecological Modelling, 133:46-56, 2000.

[18] W.M. Haddad and V. Chellaboina. Stability theory for nonnegative and compartmental dynamical systems with time delay. Syst. Control Letters, 51:355-361, 2004.

[19] L. Jaulin. Nonlinear bounded-error state estimation of continuous time systems. Automatica, 38(2):1079-1082, 2002.

[20] M. Kieffer and E. Walter. Guaranteed nonlinear state estimator for cooperative systems. Numerical Algorithms, 37:187-198, 2004.

[21] Kun Liu and Emilia Fridman. Delay-dependent methods and the first delay interval. Systems \& Control Letters, 64:57-63, 2014.

[22] F. Mazenc and O. Bernard. Interval observers for linear timeinvariant systems with disturbances. Automatica, 47(1):140$147,2011$. 
[23] F. Mazenc, S. Niculescu, and O. Bernard. Exponentially stable interval observers for linear systems with delay. SIAM J. Control Optim., 50(1):286-305, 2012.

[24] M. Moisan, O. Bernard, and J.L. Gouzé. Near optimal interval observers bundle for uncertain bio-reactors. Automatica, 45(1):291-295, 2009.

[25] P.G. Park, J. Ko, and C. Jeong. Reciprocally convex approach to stability of systems with time-varying delays. Automatica, 47:235-238, 2011.

[26] A. Polyakov, D. Efimov, W. Perruquetti, and J.-P. Richard. Output stabilization of time-varying input delay systems using interval observation technique. Automatica, 49(11):3402-3410, 2013.

[27] T. Raïssi, D. Efimov, and A. Zolghadri. Interval state estimation for a class of nonlinear systems. IEEE Trans. Automatic Control, 57(1):260-265, 2012.

[28] Mustapha Ait Rami, Michael Schnlein, and Jens Jordan. Estimation of linear positive systems with unknown timevarying delays. European Journal of Control, 19:179-187, 2013.

[29] A. Rapaport and J.L. Gouzé. Parallelotopic and practical observers for nonlinear uncertain systems. Int. J. Control, 76(3):237-251, 2003.

[30] J.-P. Richard. Time delay systems: an overview of some recent advances and open problems. Automatica, 39(10):1667-1694, 2003.

[31] R. Sipahi, S.-I. Niculescu, C. Abdallah, W. Michiels, and K. Gu. Stability and stabilization of systems with time delay limitations and opportunities. IEEE Control Systems Magazine, 31(1):38-65, 2011.

[32] H.L. Smith. Monotone Dynamical Systems: An Introduction to the Theory of Competitive and Cooperative Systems, volume 41 of Surveys and Monographs. AMS, Providence, 1995.

[33] G. Zheng, J.-P. Barbot, D. Boutat, T. Floquet, and J.-P. Richard. On observation of time-delay systems with unknown inputs. IEEE Trans. Automatic Control, 56(8):1973-1978, 2011 